\title{
Effects of family integrated care on refractory cancer-related pain receiving intrathecal morphine pump therapy: a cohort study
}

\author{
Yan Cheng", Lina Wang", Ziying Zha, Xiangqin Zhao \\ A6 East in Department of Anesthesiology, the First Affiliated Hospital of Soochow University, Suzhou, China \\ Contributions: (I) Conception and design: Y Cheng, L Wang, X Zhao; (II) Administrative support: X Zhao; (III) Provision of study materials or \\ patients: Y Cheng, L Wang, X Zhao; (IV) Collection and assembly of data: Y Cheng, L Wang; (V) Data analysis and interpretation: Y Cheng, L \\ Wang; (VI) Manuscript writing: All authors; (VII) Final approval of manuscript: All authors. \\ \#These authors contributed equally to this work. \\ Correspondence to: Xiangqin Zhao. A6 East in Department of Anesthesiology, the First Affiliated Hospital of Soochow University, No. 899 Pinghai \\ Road in Gusu District, Suzhou, China. Email: zhaoxiangqin2019@163.com.
}

Background: Family integrated care (FIC), originating from the neonatal intensive care unit, has proved
to be an effective therapy for the care of several diseases in adults. However, it is still unclear whether it is
successful in the treatment of refractory cancer-related pain using an intrathecal morphine pump (IMP).
Methods: A prospective study was performed to enroll patients with refractory cancer-related pain
treated by IMP. Medical records, including demographic data, types of cancer, doses of opioids before the
implantation, were gathered at baseline. Besides, numerical pain rating scales (NRS) were measured to assess
the severity of pain. Karnofsky performance scores (KPS), self-rating anxiety scale (SAS) scores and self-
rating depression scale (SDS) scores were used to assess the physical and psychological status of patients.
Follow-up lasted for at least 12 months. The changes in NRS, KPS, SAS, and SDS scores were recorded, as
well as adverse events and survival rates. Results: Totally, 33 patients were enrolled and divided into traditional care groups and FIC groups. The results showed that the implantation of IMP relieved the pain in both groups. Moreover, patients in the FIC group had significantly more relief of pain than those in the traditional group ( $\mathrm{P}=0.04)$. Similarly, KPS raised more, and scores of SAS and SDS decreased more in the FIC group. Besides, fewer adverse events occurred in the FIC group, but the survival rate showed no significant difference between the two groups.

Conclusions: FIC enhances the effects of IMP on the relief of refractory cancer-related pain and improves the physical and psychological status of patients.

Keywords: Family integrated care (FIC); intrathecal morphine pump (IMP); refractory cancer pain; pain relief; survival rate; adverse events

Submitted May 22, 2020. Accepted for publication Jul 10, 2020.

doi: 10.21037/apm-20-1293

View this article at: http://dx.doi.org/10.21037/apm-20-1293

\section{Introduction}

As reported, the 5 -year survival rate of patients with all kinds of cancers has increased to $65 \%$ (1). Meanwhile, many survival patients are suffering from chronic cancerrelated pain. It has been reported that the prevalence of chronic cancer-related pain reaches $70-90 \%$ in the advanced stage of cancer (2), which needs long-term use of opioids for pain relief. The three-step analgesic ladder therapy recommended by the World Health Organization (WHO) helps improve the chronic pain in over $80 \%$ of cases (3). However, the therapy is still to do not relieve pain in $15-20 \%$ of cases due to drug tolerance or serious adverse events. To make up for the shortcomings of three-step analgesic ladder therapy, the novel, a fourth step, including several therapies, was put forward (4), of which intrathecal drug infusion was most discussed. 
Intrathecal infusion of opioids is designed to directly deliver drugs into the cerebrospinal fluid to bind to their spinal receptors (5). It has been reported that delivering morphine at $1 / 300$ of oral dose via intrathecal infusion supplies a strong analgesic effect and reduces the incidence of the adverse events caused by systemic opioids (6). There are two types of intrathecal infusion, either using an implanted morphine pump or a percutaneous port $(7,8)$. Implanted intrathecal morphine pump (IMP) costs more than a percutaneous port, but it supplies a more stable administration rate and a better quality of life. Therefore, IMP has been widely used in patients with refractory cancer-related pain in recent years.

In addition to the novel therapy, nursing care also plays a crucial role in the treatment of refractory cancer-related pain. The model of traditional nursing care is dominated by medical staff, and it supplies professional treatment advice for patients in hospitals. Nevertheless, this model played is inferior for patients discharged from the hospital. The family integrated care (FIC) model appears, along with the development of nursing care. FIC was initially designed to incorporate parents into the nursing care department in the neonatal intensive care unit to improve the growth of their infants $(9,10)$. Afterward, some attempts have been made to explore the effects of FIC on adult patients, including patients with intellectual disability, acquired immune deficiency syndrome, and dementia (11-13). Their results showed that patients treated with the FIC model received a better prognosis than those treated with traditional care. However, it is still unknown whether FIC is successful in the treatment of refractory cancer-related pain using IMP. The purpose of the present study is to determine the effects of FIC on patients with refractory cancer pain receiving IMP therapy. We present the following article in accordance with the STROBE reporting checklist (available at http:// dx.doi.org/10.21037/apm-20-1293).

\section{Methods}

\section{Patient population}

From January 2017 to June 2018, all adult patients with refractory cancer-related pain, who were admitted to the department of anesthesiology of the first affiliated hospital of Soochow University, were enrolled in the present study. The following patients were excluded: those who did not take morphine as an analgesic underwent a surgical or invasive procedure in recent three months, have been enrolled in any other drug or device study which may bias our study or be out of contact during follow-up. All enrolled patients signed informed consent before data collection. The study was performed in compliance with the ethical principles of the Declaration of Helsinki (as revised in 2013) and has been approved by the ethics committee of the First Affiliated Hospital of Soochow University.

The implantation of IMP was performed by skilled surgeons. Briefly, patients were anesthetized locally, and then surgeons punctured the corresponding spinal segment according to the pain site. Under the guidance of $\mathrm{X}$-ray fluoroscopy, the drug delivery catheter was inserted into the subarachnoid cavity of the spinal segment. The morphine pump was then connected with the drug delivery catheter and inserted into abdominal subcutaneous tissue. Morphine was added into the morphine pump, and parameters of morphine infusion were set at $1 / 300$ of the oral dose. After the surgical incision was healed, and the dose of morphine was stable, patients were discharged from the hospital. Routine treatment of cancer, including systemic chemotherapy, radiotherapy, molecule-targeted therapy, or symptomatic and supportive treatments, would continue after discharge.

\section{Data gathering procedure}

When implanted with IMP and enrolled in the present study, medical records of patients were prospectively gathered, including demographic data, types of cancer, doses of opioids before the implantation. Besides, numerical pain rating scales (NRS) are used to measure the severity of pain in patient ratings from 0 to 10 scores. Karnofsky performance scores (KPS) was used to measure the physical status of patients rating from 0 to 100 scores. Also, selfrating anxiety scale (SAS) scores and self-rating depression scale (SDS) scores were used to assess the psychological status of patients.

\section{Interventions}

All enrolled patients received traditional nursing care in hospitals. Additionally, some family members of patients received professional training of nursing care on their willingness, which included psychological intervention, health education, medication care, and living care. Also, they can take part in decision-making and share information with medical staff. Patients are then divided into two groups according to whether their family members received 
Table 1 Demographic and clinical data of enrolled patients

\begin{tabular}{|c|c|c|c|c|}
\hline Parameters & Total & Traditional group & FIC group & $P$ value \\
\hline Age & $58.64 \pm 7.04$ & $59.74 \pm 7.42$ & $57.14 \pm 6.43$ & 0.30 \\
\hline Gender (M/F) & $21(63.63 \%) / 12(36.36 \%)$ & 12 (63.16\%)/7 (36.84\%) & $9(64.29 \%) / 5(35.71 \%)$ & 0.95 \\
\hline $\mathrm{BMI}\left(\mathrm{kg} / \mathrm{m}^{2}\right)$ & $20.03 \pm 3.45$ & $20.49 \pm 3.48$ & $19.38 \pm 3.42$ & 0.37 \\
\hline \multicolumn{5}{|l|}{ Cancer types, n (\%) } \\
\hline Lung cancer & $9(27.27)$ & $7(36.84)$ & $2(14.29)$ & 0.15 \\
\hline Gastrointestinal cancer & $7(21.21)$ & $4(21.05)$ & $3(21.43)$ & 0.98 \\
\hline Hepatobiliary cancer & $5(15.15)$ & $4(21.05)$ & $1(7.14)$ & 0.27 \\
\hline Gynecological cancer & $3(9.09)$ & $0(0)$ & $3(21.43)$ & 0.04 \\
\hline Other & $2(6.06)$ & $1(5.26)$ & $1(7.14)$ & 0.82 \\
\hline NRS score before implantation & $7.76 \pm 1.15$ & $7.58 \pm 1.22$ & $8.00 \pm 1.04$ & 0.31 \\
\hline KPS before implantation & $72.27 \pm 10.66$ & $70.89 \pm 9.78$ & $74.14 \pm 11.87$ & 0.40 \\
\hline SAS score before implantation & $57.91 \pm 11.82$ & $55.63 \pm 12.22$ & $61.00 \pm 10.91$ & 0.20 \\
\hline SDS score before implantation & $62.12 \pm 11.64$ & $60.47 \pm 12.68$ & $64.36 \pm 10.07$ & 0.35 \\
\hline
\end{tabular}

M, male; F, female; BMI, body mass index; NRS, numerical pain rating scales; KPS, Karnofsky performance scores; SAS, self-rating anxiety scale; SDS, self-rating depression scale.

training, one is the FIC group (14 patients), and the other is the traditional care group (19 patients).

\section{Outcomes}

The follow-up in the present study lasted at least one year. The primary outcome is the change of NRS scores. The NRS score was measured at each visit after implantation and recorded when it reached a stable status. The secondary outcomes are the change of KPS, SAS and SDS scores, adverse events, and the survival rate of patients. The adverse events included severe adverse events, including systematic infection, psychiatric disorders, respiratory disorders, and poisoning complications, and mild adverse events, including nausea, vomiting, dizziness, diarrhea, skin reactions, hypotension, constipation, drowsiness, and headache.

\section{Data analysis}

All data in the present study were analyzed by SPSS (v. 20.0,
IBM Corp., Armonk, NY, USA). Continuous variables were reported as means with standard deviations, and categorical variables were expressed as percentages. Comparisons between the two groups were performed using a 2-tailed $t$-test for continuous variables and the $\chi^{2}$ test for categorical variables. Survival curves were generated using the KaplanMeier method, and the log-rank test was performed to compare the difference between the two groups. The significant level was set as $\mathrm{P}<0.05$.

\section{Results}

\section{Patient baseline data}

We prospectively collected data from 33 patients with refractory cancer-related pain in the present study. Their demographic and clinical data are listed in Table 1. The average age of enrolled patients was $58.64 \pm 7.04$ years old, and there were 21 male and 12 female patients, respectively. Average BMI was $20.03 \pm 3.45 \mathrm{~kg} / \mathrm{m}^{2}$, and chronic cancer-related pain has lasted for an average duration of 
$0.58 \pm 0.38$ years. The types of cancer in enrolled patients were diverse. Nine patients have lung cancer, 7 patients who have gastrointestinal cancer, 5 patients who have hepatobiliary cancer, 4 patients who have pancreatic cancer, 3 patients who have breast cancer, 3 patients who have gynecological cancer, and 2 patients who have other types of cancer, respectively. The level of pain in patients before the implantation of IMP was assessed by NRS, an average score of which was $7.76 \pm 1.15$ points. Besides, KPS, SAS, and SDS were used to assess physical and psychological status before the implantation. As shown in Table 1, the average KPS was $72.27 \pm 10.66$ points, the average score of SAS was $57.91 \pm 11.82$ points, and the average score of SDS was $62.12 \pm 11.64$ points.

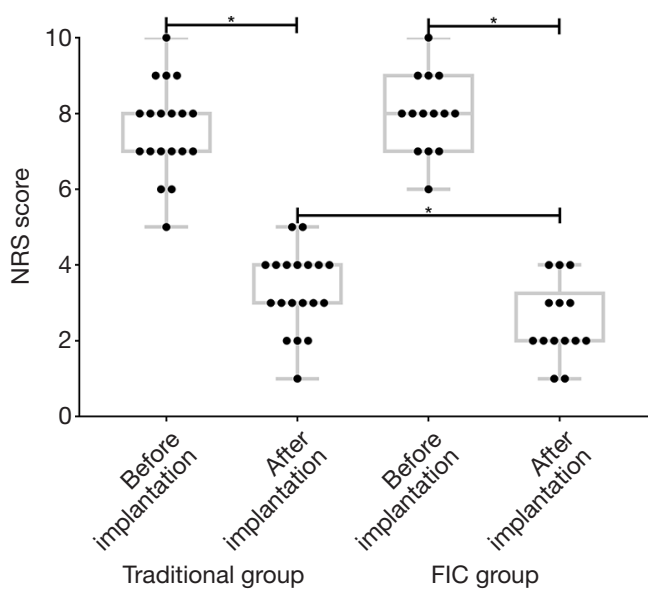

Figure 1 Comparison of NRS score before and after implantation in traditional and FIC groups. *, $\mathrm{P}<0.05$. NRS, numerical pain rating scales; FIC, family integrated care.
Then we divided enrolled patients into two groups, traditional care group and FIC group. The traditional group had 19 patients, and the FIC group had 14 patients, as shown in Table 1. According to the comparison between the two groups, we found there was no significant difference between them in most parameters, except that the FIC group had 3 patients who have gynecological cancer while the traditional group had none $(\mathrm{P}=0.04)$.

\section{Improvement of refractory cancer-related pain by FIC}

All enrolled patients were followed up for at least 12 months. We recorded NRS, KPS, SAS, and SDS at each visit. The stable NRS scores of patients in both groups were shown in Figure 1. We found that the implantation of IMP could improve refractory cancer-related pain in both groups $(\mathrm{P}<0.001)$. Moreover, we found that the average score of NRS after implantation in the FIC group was much lower than that in the traditional group $(\mathrm{P}=0.03)$, showing a better analgesic effect. The changes of KPS, SAS, and SDS scores before and after implantation in both groups were shown in Figure 2. The results were the result of the change of NRS scores. The implantation of IMP raised KPS and reduced the score of SAS and SDS, showing an improvement of physical and psychological status in patients $(\mathrm{P}<0.001)$. Patients in the FIC group had a more significant increase of KPS and a more significant decrease of SAS and SDS scores than those in the traditional groups $(\mathrm{P}=0.03,0.02$, and 0.03 , respectively).

\section{Improvement of adverse events by FIC}

Adverse events that occurred during the follow-up were
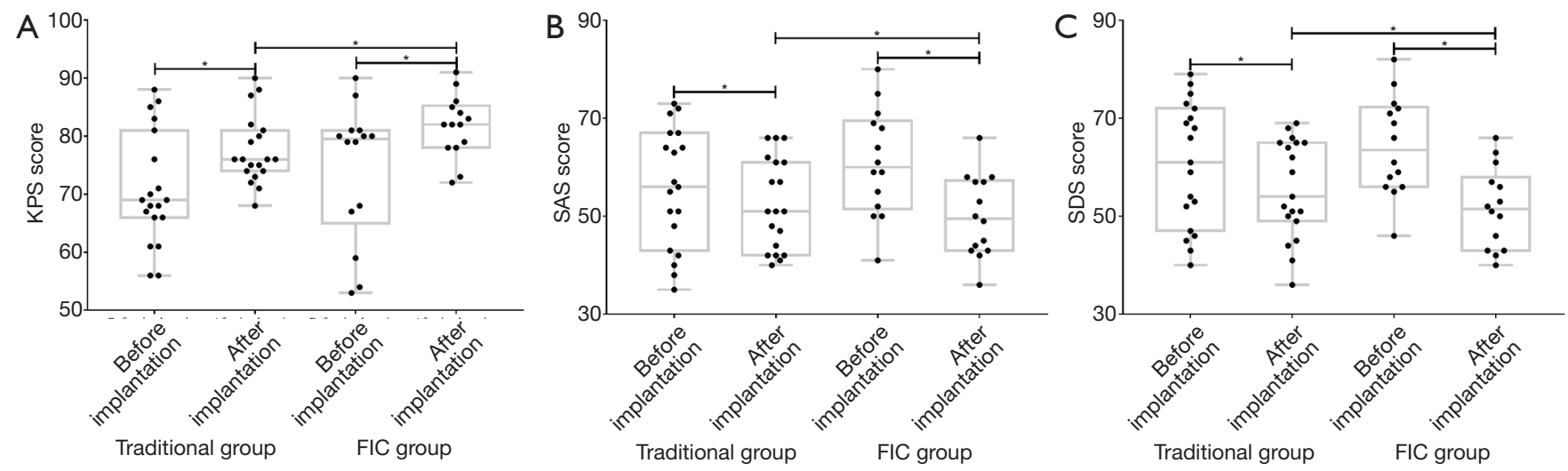

Figure 2 Comparison of KPS, SAS and SDS score before and after implantation in traditional and FIC groups. *, P<0.05. KPS, Karnofsky performance scores; SAS, SAS, self-rating anxiety scale; SDS, self-rating depression scale; FIC, family integrated care. 
Table 2 Adverse events occurred in enrolled patients

\begin{tabular}{lccc}
\hline Parameters & Total & Traditional group & FIC group \\
\hline Overall cases $^{*}$ & 34 & 22 & 12 \\
Nausea/vomiting & 11 & 8 & 3 \\
Drowsiness & 8 & 6 & 2 \\
Constipation & 5 & 4 & 1 \\
Dizziness & 4 & 2 & 2 \\
Skin reactions & 3 & 1 & 2 \\
Hypotension & 2 & 0 & 2 \\
Diarrhea & 1 & 1 & 0 \\
\hline
\end{tabular}

*, each patient may suffer from more than one adverse event.

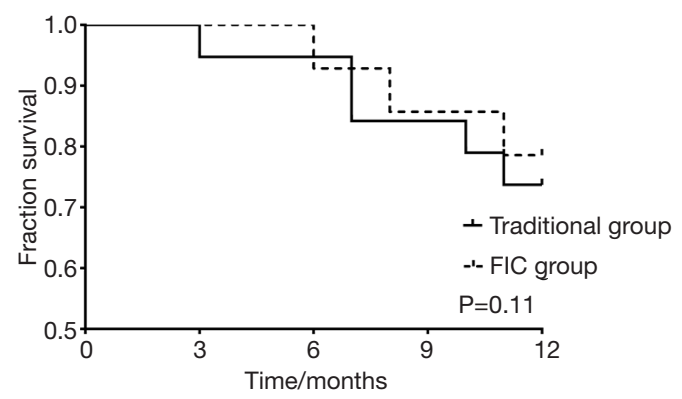

Figure 3 Comparison of patient survival rate from all-cause mortality in traditional and FIC groups using log-rank test. FIC, family integrated care.

also recorded. No serious adverse events occurred in the present study, and some mild adverse events are listed in Table 2. Thirty-four adverse events occurred in the present study, and 15 patients suffering from more than one adverse event. As shown in Table 2, the most common adverse event was nausea/vomiting, which occurred in 8 cases in the traditional group and 3 cases in the FIC group. Drowsiness was common as well, and it occurred in 6 cases in the traditional group and 2 cases in the FIC group. Some other adverse events also occurred, including constipation, dizziness, skin reactions, hypotension, and diarrhea, as listed in Table 2. In general, FIC groups had 12 cases of an adverse event in total, which was fewer than 22 cases in the traditional group.

There were 5 patients dead in a traditional group, and 3 patients died in the FIC group during follow-up, for which we then performed a survival curve analysis (Figure 3). The results indicated that there was no significant difference between traditional and FIC groups, comparing the survival rate of enrolled patients $(\mathrm{P}=0.11)$.

\section{Discussion}

To our knowledge, this is the first study that applied FIC into the nursing care of patients with refractory cancerrelated pain treated with IMP. Earlier studies have confirmed that FIC provided a better prognosis to patients with intellectual disability, acquired immune deficiency syndrome, and dementia (11-13). Similarly, our study also confirmed FIC was of significant benefit to patients with refractory cancer-related pain treated with IMP, by enhancing the relief of pain, improving the physical and psychological status of patients and reducing the incidence of adverse events.

Implantation of IMP is the last choice for patients suffering from refractory cancer-related pain. In practice, many studies have revealed the significant effects of IMP on pain relief $(8,14-16)$. Meanwhile, IMP was reported to induce the improvement of physical status, like the results in our study (17). Psychological status is a research focus as well. Cancer survivors may experience pain, fatigue, or psychological distress during treatment, which would affect their ability to work and their prognosis $(18,19)$. Li et al. have reported a direct relationship between cancerrelated pain and psychological distress, in which patients suffering from pain were more prone to anxiety and depression (20). A systematic review has reported that intrathecal drug infusion is beneficial to the improvement of psychological status in patients with cancer-related or non-cancer-related pain (21). Our study used SAS and SDS scores to assess the levels of anxiety and depression in patients and found that anxiety and depression indeed were alleviated by IMP.

The FIC enables family members of patients to learn more knowledge about nursing care of IMP and the use of morphine. Additionally, they were informed of the importance of psychological counseling. The model provides better nursing care for patients, especially after discharge. In our study, patients in the FIC group had a lower level of NRS score than those in the traditional care group. It can be explained that patient families in the FIC group paid more attention to the nursing care of patients and communicated more with patients. Therefore, deficiencies in drug doses can be found and adjusted earlier. More relief of pain leads to better physical and psychological status in patients in the FIC group, shown by higher KPS and lower scores of SAS and 
SDS scores in our study.

The intrathecal opioid infusion usually induces an insufficient number of adverse events and seldom induces serious adverse events $(14,22,23)$. Our study only observed some mild adverse events in the same way, including nausea, vomiting, dizziness, diarrhea, skin reactions, hypotension, constipation, and drowsiness. In general, the occurrence of mild adverse events in our study had no significant impacts on the prognosis and daily life of patients. FIC model also played an essential role in the reduction of adverse events in our study. It was found that fewer adverse events occurred in the FIC group than the traditional group in our study. The primary reason is that FIC encourages family members of patients to carefully record the response of patients after receiving drug infusion through IMP. Adverse events are managed in time by adjusting the dose of morphine or giving symptomatic treatment to prevent the aggravation of symptoms. In the traditional group, adverse events are challenging handling appropriately due to insufficient professional knowledge in family members of patients, which may lead to more adverse events.

Determining the survival of enrolled patients is one of the primary purposes of our study. Unfortunately, FIC showed no significant effects on the survival of patients. For most patients with advanced cancer, risk factors of prognosis are reported to be age, metastasis and drug susceptibility (24-26). However, Trautmann et al. found that colon cancer care with evidence-based quality standards has positive effects on patient outcomes (27). There were 5 patients dead in a traditional group, and 3 patients died in the FIC group, which indicates lower mortality in the FIC group, but many patients limited further analysis.

There were several limitations to the present study. The first is the number of enrolled patients that were inadequate due to the prohibitive cost of IMP implantation. The second is that the duration of follow-up is not extensive enough, considering the 5 -year survival rate is increasing nowadays. A more significant number of patients and a longer follow-up may allow us to explore further the effects of FIC on adverse events and survival rates of patients. Besides, the results obtained from a study focusing on patients with specific types of cancer may be more helpful in guiding the nursing care of refractory cancer-related pain. Our study supplies a novel insight into FIC, but more studies are still to be performed in the future.

In conclusion, the implantation of IMP is an effective therapy for refractory cancer-related pain, which reliefs the pain and induces no serious adverse events. FIC, as a novel attempt in nursing care, further enhances the effects of IMP on refractory cancer-related pain and improves the physical and psychological status of patients. Moreover, it reduces the incidence of mild adverse events induced by morphine to some degree. The effects of FIC on the survival of patients are still to be explored further.

\section{Acknowledgments}

Funding: The Jiangsu Provincial Medical Youth Talents Program supports this work (QNRC2016740) and the National Natural Science Foundation of China (81701098).

\section{Footnote}

Reporting Checklist: The authors have completed the STROBE reporting checklist. Available at http://dx.doi. org/10.21037/apm-20-1293

Data Sharing Statement: Available at http://dx.doi. org/10.21037/apm-20-1293

Conflicts of Interest: All authors have completed the ICMJE uniform disclosure form (available at http://dx.doi. org/10.21037/apm-20-1293). The authors have no conflicts of interest to declare.

Ethical Statement: The authors are accountable for all aspects of the work in ensuring that questions related to the accuracy or integrity of any part of the work are appropriately investigated and resolved. All enrolled patients signed informed consent before data collection. The study was performed in compliance with the ethical principles of the Declaration of Helsinki (as revised in 2013) and has been approved by the ethics committee of the First Affiliated Hospital of Soochow University (approval ID: 2120160022).

Open Access Statement: This is an Open Access article distributed in accordance with the Creative Commons Attribution-NonCommercial-NoDerivs 4.0 International License (CC BY-NC-ND 4.0), which permits the noncommercial replication and distribution of the article with the strict proviso that no changes or edits are made and the original work is properly cited (including links to both the formal publication through the relevant DOI and the license). See: https://creativecommons.org/licenses/by-nc$\mathrm{nd} / 4.0 \%$. 


\section{References}

1. Miller KD, Nogueira L, Mariotto AB, et al. Cancer treatment and survivorship statistics, 2019. CA Cancer J Clin 2019;69:363-85.

2. Soares LG. The pain watch: it's about time to relieve cancer pain. Ann Palliat Med 2019;8:523-4.

3. Meuser T, Pietruck C, Radbruch L, et al. Symptoms during cancer pain treatment following WHO-guidelines: a longitudinal follow-up study of symptom prevalence, severity and etiology. Pain 2001;93:247-57.

4. Miguel R. Interventional treatment of cancer pain: the fourth step in the World Health Organization analgesic ladder? Cancer Control 2000;7:149-56.

5. Mercadante S, Porzio G, Gebbia V. Spinal analgesia for advanced cancer patients: an update. Crit Rev Oncol Hematol 2012;82:227-32.

6. Bhatia G, Lau ME, Koury KM, et al. Intrathecal Drug Delivery (ITDD) systems for cancer pain. F1000Res 2013;2:96.

7. Brogan SE. Intrathecal therapy for the management of cancer pain. Curr Pain Headache Rep 2006;10:254-9.

8. Puntillo F, Giglio M, Preziosa A, et al. Triple intrathecal combination therapy for end-stage cancer-related refractory pain: a prospective observational study with two-month follow-up. Pain Ther 2020. [Epub ahead of print].

9. O'Brien K, Robson K, Bracht M, et al. Effectiveness of Family Integrated Care in neonatal intensive care units on infant and parent outcomes: a multicentre, multinational, cluster-randomised controlled trial. Lancet Child Adolesc Health 2018;2:245-54.

10. O'Brien K, Bracht M, Robson K, et al. Evaluation of the Family Integrated Care model of neonatal intensive care: a cluster randomized controlled trial in Canada and Australia. BMC Pediatr 2015;15:210.

11. Onrust SA, Romijn G, de Beer Y. Family Group Conferences within the integrated care system for young people with ID: a controlled study of effects and costs. BMC Health Serv Res 2015;15:392.

12. Kanyangarara M, Sakyi K, Laar A. Availability of integrated family planning services in HIV care and support sites in sub-Saharan Africa: a secondary analysis of national health facility surveys. Reprod Health 2019;16:60.

13. Robertshaw D, Cross A. Experiences of integrated care for dementia from family and carer perspectives: a framework analysis of massive open online course discussion board posts. Dementia 2019;18:1492-506.
14. Liu W, Liu H, Zhang Z, et al. Effects of intraperitoneal and intrathecal morphine analgesia on the expression of mu-opioid receptors in bone cancer pain rats. Dose Response 2019;17:1559325819882873.

15. Dupoiron D, Leblanc D, Demelliez-Merceron S, et al. Optimizing Initial Intrathecal Drug Ratio for Refractory Cancer-Related Pain for Early Pain Relief. A Retrospective Monocentric Study. Pain Med 2019;20:2033-42.

16. Carvajal G, Dupoiron D, Seegers V, et al. Intrathecal Drug Delivery Systems for Refractory Pancreatic Cancer Pain: Observational Follow-up Study Over an 11-Year Period in a Comprehensive Cancer Center. Anesth Analg 2018;126:2038-46.

17. Qin W, Li Y, Liu B, et al. Intrathecal Morphine Infusion Therapy via a Percutaneous Port for Refractory Cancer Pain in China: An Efficacy, Safety and Cost Utilization Analysis. J Pain Res 2020;13:231-7.

18. Bjerkeset E, Rohrl K, Schou-Bredal I. Symptom cluster of pain, fatigue, and psychological distress in breast cancer survivors: prevalence and characteristics. Breast Cancer Res Treat 2020;180:63-71.

19. McCowat M, Fleming L, Vibholm J, et al. The Psychological Predictors of Acute and Chronic Pain in Women Following Breast Cancer Surgery: A Systematic Review. Clin J Pain 2019;35:261-71.

20. Li XM, Xiao WH, Yang P, et al. Psychological distress and cancer pain: Results from a controlled cross-sectional survey in China. Sci Rep 2017;7:39397.

21. Hayek SM, Deer TR, Pope JE, et al. Intrathecal therapy for cancer and non-cancer pain. Pain Physician 2011;14:219-48.

22. Kim EK, Shin JY, Castaneda AM, et al. Retrospective analysis of the financial break-even point for intrathecal morphine pump use in Korea. Korean J Pain 2017;30:272-80.

23. Mastenbroek TC, Kramp-Hendriks BJ, Kallewaard JW, et al. Multimodal intrathecal analgesia in refractory cancer pain. Scand J Pain 2017;14:39-43.

24. Junjun L, Pei W, Ying Y, et al. Prognosis and risk factors in older patients with lung cancer and pulmonary embolism: a propensity score matching analysis. Sci Rep 2020;10:1272.

25. Wang X, Huang J, Bingbing Z, et al. Risk factors, risk assessment, and prognosis in patients with gynecological cancer and thromboembolism. J Int Med Res 2019. [Epub ahead of print].

26. Sorgun MH, Kuzu M, Ozer IS, et al. Risk Factors, Biomarkers, Etiology, Outcome and Prognosis of Ischemic 
Stroke in Cancer Patients. Asian Pac J Cancer Prev 2018;19:649-53.

27. Trautmann F, Reissfelder C, Pecqueux M, et al. Evidencebased quality standards improve prognosis in colon cancer

Cite this article as: Cheng Y, Wang L, Zha Z, Zhao X. Effects of family integrated care on refractory cancer-related pain receiving intrathecal morphine pump therapy: a cohort study. Ann Palliat Med 2020;9(4):2163-2170. doi: 10.21037/apm-20-1293 care. Eur J Surg Oncol 2018;44:1324-30.

(English Language Editor: J. Chapnick) 